\title{
Wie kontrolliert man Gesundheitskosten?
}

\author{
Wie behält man die Gesundheitskosten im Griff? Zu diesem Thema sprachen fünf \\ Referentinnen und Referenten an einer vom Zürcher Institut Dialog Ethik organisier- \\ ten Veranstaltung in Bern zum Thema «Organisation der Kostenkontrolle im KVG».
}

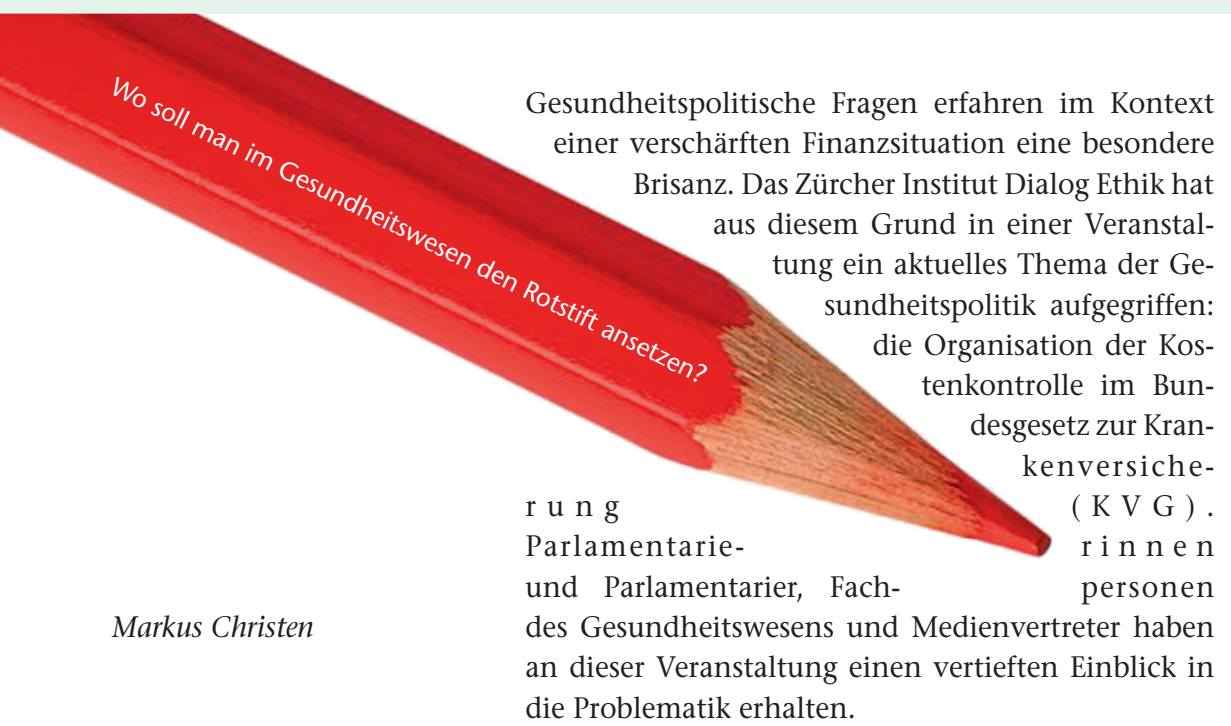

\section{Geschundenes Versicherungsprinzip}

Nach den Begrüssungsworten von Gastgeber Hansueli Mösle, dem Direktor von Curaviva Schweiz, hat der Jurist Max Baumann in die Thematik eingeführt. Er erinnerte daran, dass das KVG von Parlamentarierinnen und Parlamentariern geschaffen worden war, welche die enormen Fortschritte der Medizin zwischen 1945 und 1995 am eigenen Leib erlebt haben und deshalb bei der Schaffung des Bundesgesetzes von einem stetigen Fortschritt in der Medizin ausgegangen waren. Doch heute sei der medizinische Fortschritt geringer und die Kosten dafür grösser, so dass eine Kontrolle der Kosten unumgänglich sei. Mehrere Wahrnehmungs-

\section{Kontrolle von Kosten kann keine primäre ärztliche Aufgabe sein}

lücken verunmöglichten es derzeit aber, die Gesundheitskosten «in der Hand zu haben». So sei es erstens erstaunlich, wie ungleichmässig Forschungsressourcen verteilt sind: Während enorm viele Gelder für Forschungen im Bereich Spitzenmedizin gebraucht würden, interessiere sich kaum jemand für krankheitsrelevante Fragen ausserhalb der Klinik.

Zweitens sorge die derzeitige «Innovations-Dynamik» in der Medizin dafür, dass sich kaum mehr eine Routine für Behandlungsformen entwickeln könne. Langzeitvergleiche im Hinblick auf die Kosteneffektivität von medizinischen Neuerungen würden da- durch faktisch in vielen Fällen verunmöglicht. Drittens schliesslich werde das KVG von vielen gar nicht mehr als eine Versicherung verstanden. Prämienzahler gingen davon aus, dass sie ein Anrecht darauf hätten, mindestens soviel von der Krankenversicherung zurückzubekommen, wie sie einbezahlt hätten. Die Leistungserbringer hauen in die gleiche Kerbe und regen zum Konsum von medizinischen Dienstleistungen an - schliesslich hätten die Kunden dafür ja via Prämie bezahlt. Doch damit werde das Versicherungsprinzip mit Füssen getreten. Mögliche Gegenmassnahmen seien gemäss Baumann unter anderem eine Erhöhung der direkten Selbstbeteiligung (wobei im Gegenzug die Prämien gesenkt werden könnten) sowie eine klarere Definition, welche Leistungen durch das KVG bezahlt würden.

\section{Sind die Politiker schuld?}

Ständerätin Christine Egerszegi-Obrist ging in ihren Ausführungen auf die Rolle der Politik in der Kostenkontrolle im Gesundheitswesen ein. Es sei zu einfach, die Politik für die Kostensteigerung verantwortlich zu machen. So könne das Parlament lediglich den politischen Rahmen festlegen, der Platz lassen müsse für neue Entwicklungen. Es sei auch falsch anzunehmen, dass das KVG Ursache der Kostensteigerung sei. Vielmehr habe der Souverän in mehreren Abstimmungen jeweils für einen Ausbau der solidarisch getragenen Gesundheitsleistungen plädiert. Zudem sei das Gesundheitswesen eng mit anderen gesellschaftlichen Bereichen vernetzt. So dürfte beispielsweise die Einsparung des Hauswirtschaftsunterrichts mit dazu beigetragen haben, dass es immer mehr übergewichtige Kinder gebe, für die nun im Gesundheitswesen wiederum künftig Kosten anfallen. Diese enge Verflechtung gesundheitspolitischer Fragen mit anderen gesellschaftlichen Prozessen mache eine Kostenkontrolle schwierig. Heute sei davon auszugehen, dass jeder Eingriff zur Senkung der Gesundheitskosten immer auch Verlierer produziere. Dies erkläre auch, warum der politische Prozess in diesem Bereich langsam und schwierig ist.

FMH-Präsident Jacques de Haller verdeutlichte, dass die Kontrolle von Kosten keine primäre ärztliche Aufgabe sein könne. Der Mediziner richte sich an den einzelnen Menschen, doch die Kostenkontrolle verlange eine umfassende, gleichsam vereinheitlichende 
Sicht auf alle Prozesse in der Medizin. Dies erzeuge ein Spannungsfeld, das man nicht einfach verdrängen könne. Da das Gesundheitssystem in erster Linie durch die Tätigkeit von Menschen geprägt ist, bedeutet Kostenkontrolle nichts anderes, als dass der «Faktor Mensch» zurückgebunden werden müsse - beispielsweise dadurch, indem weniger Menschen im Gesundheitswesen arbeiten oder weniger Zeit für den Kontakt zwischen Arzt und Patient zur Verfügung steht. Man könne demnach nicht so tun, als ob Kostenkontrolle eine rein medizinische Frage sei - etwa erreichbar durch eine kosteneffektivere Behandlungspraxis. Sie greift ein in die Ausgestaltung der Beziehung zwischen Arzt und Patient, was einer ethischen, nicht aber medizinischen Rechtfertigung bedarf. sicherung gegen Krankheit zu erreichen, sei eine Einschränkung der solidarisch finanzierten Leistungen unumgänglich. Dabei fänden sich vier Positionen zur Frage, wie man die Kosten im Gesundheitswesen wieder in den Griff bekommen könnte. Marktvertreter setzen auf eine Verstärkung des Marktes im Gesundheitswesen und damit verbunden auf eine «Selbstrationierung» durch die Betroffenen selbst. Knappheitsskeptiker bestreiten die Notwendigkeit der Einschränkung wichtiger Leistungen - es würde reichen, «unnütze» Leistungen nicht mehr solidarisch zu finanzieren. Vertreter der Position «Gesundheit hat keinen Preis» sind der Ansicht, es müssten andere solidarisch finanzierte Bereiche zulasten des Gesundheitswesens weniger Geld bekommen. Rationierer schliess-

\section{In einer Wohlstandsgesellschaft erhält Gesundheit ein immer höheres Gewicht und die Menschen investieren dafür mehr Zeit, Geld und Aufmerksamkeit}

\section{Ein überbelastetes KVG?}

Peter Indra, Vizedirektor des BAG und Leiter der Abteilung KVG/UVG, verwies in seinen Ausführungen auf die Tendenz, möglichst alle gesundheitspolitischen Themen im Rahmen des KVG abhandeln zu wollen und dann letztlich auch im Rahmen des KVG finanzieren zu wollen. Ein Beispiel ist die Prävention, die nicht zu den Zielsetzungen des KVG gehöre und für die nun glücklicherweise bald ein eigenes Gesetz geschaffen werde. Auch werde es immer schwieriger, die KVG-Kriterien für eine qualitativ gute Gesundheitsversorgung - Wirksamkeit, Zweckmässigkeit und Wirtschaftlichkeit (WZW) - faktisch zu prüfen. So gebe es Medikamente, welche die durchschnittliche Mobilität von Patienten zum Preis von einer halben Million pro Fall von 350 auf 380 Meter erhöhe - eine statistisch signifikante Verbesserung, doch kann man dies noch als zweckmässig bezeichnen? Indra machte jedenfalls deutlich, dass eine Kostenkontrolle im KVG notwendig ist, um das Versicherungsobligatorium, die Solidarität zwischen Gesunden und Kranken sowie den freien Zugang zu einem definierten Leistungspaket weiter gewährleisten zu können. Hingegen müsse das KVG keine Strukturerhaltung im Gesundheitswesen gewährleisten.

Der Medizinethiker Markus Zimmermann-Acklin von der Universität Luzern erinnerte schliesslich daran, dass in einer Wohlstandsgesellschaft Gesundheit ein immer höheres Gewicht erhalte und die Menschen mehr Zeit, Geld und Aufmerksamkeit dafür investierten. Um in einem solchen Umfeld eine gerechte Ver- lich fordern eine - entweder bewusst gesteuerte oder aber implizite - Rationierung von Gesundheitsdienstleistungen. Zimmermann-Acklin plädierte für die Position des bewussten Rationierens, ist sich aber der Probleme der praktischen Umsetzung einer Rationierung bewusst. Ein akzeptables Vorgehen könnte darin bestehen, die WZW-Kriterien für eine qualitativ gute Gesundheitsversorgung für alle enger als bisher auszulegen.

Ruth Baumann-Hölzle, Leiterin des Instituts Dialog Ethik, moderierte die anschliessende Diskussion, in der kontrovers über Solidarität und Qualität im Gesundheitswesen diskutiert wurde. Einigkeit bestand aber darin, dass der Versicherungsgedanke des KVG wieder vermehrt Beachtung finden müsse. Demnach sollte das KVG vorab dann zum Tragen kommen, wenn Menschen mit Gesundheitseinschränkungen konfrontiert seien, die sie finanziell nicht mehr selbst tragen könnten. Ein «gesundheitspolitisches Schlaraffenland» könne aber nicht durch eine solidarisch finanzierte Versicherung geschaffen werden.

Hintergrund der Veranstaltung war die Veröffentlichung des vierten Bandes «Verantwortung im politischen Diskurs» des fünfbändigen «Handbuch Ethik im Gesundheitswesen», das Dialog Ethik in Kooperation mit den Verlagen Schwabe und EMH herausgibt. Über 80 Autorinnen und Autoren aus vier europäischen Staaten haben an diesem Werk, das im gesamten deutschsprachigen Raum verbreitet wird, mitgearbeitet. 